\title{
RELAÇÕES ENTRE A SUBSTITUIÇÃO ISOMÓRFICA DE Fe POR AI E AS CARACTERÍSTICAS QUÍMICAS E MINERALÓGICAS DE HEMATITAS SINTÉTICAS(1)
}

\author{
J. A. SAMBATTI( ${ }^{(2)}$, A. C. S. COSTA ${ }^{(3)}$, A. S. MUNIZ ${ }^{(4)}$, E. SE NGIK(4), \\ I. G. SOUZA J UNIOR ${ }^{(2)} \&$ J . M. BIGHAM(5)
}

\begin{abstract}
RESUMO
Foram sintetizadas hematitas com diferentes graus de substituição isomórfica (SI) de Fe por Al até um máximo de $16 \mathrm{Al}$ mol\%. Os minerais foram caracterizados por difratometria de raios-X, análise química total, extração de ferro e alumínio solúveis em oxalato ácido de amônio $\left(\mathrm{Fe}_{\mathrm{o}}+\mathrm{Al}_{\mathrm{o}}\right)$, determinação da capacidade de troca aniônica (CTA) e área superficial específica (ASE). Os resultados das análises mostraram que os métodos de síntese resultaram em uma quantidade mínima de impurezas e que, de maneira geral, os valores de substituição isomórfica previstos foram alcançados. Os valores de ASE foram relativamente baixos para as hematitas com até $10 \%$ de $\mathrm{Al}$ e elevados para as hematitas com 13 e 16\% de Al de substituição isomórfica. Foi observada estreita correlação entre a ASE e a CTA com os teores de Fe e Al solúveis em oxalato ácido de amônio $\left(\mathrm{Fe}_{\mathrm{o}}+\mathrm{Al}_{\mathrm{o}}\right)$.
\end{abstract}

Termos de indexação: área superficial específica, CTA, DRX, óxidos de ferro.

\section{SUMMARY: RELATIONSHIPS BETWEEN THE ISOMORPHIC SUBSTITUTION OF FE FOR AL AND THE CHEMICAL AND MINERALOGICAL CHARACTERISTICS OF SYNTHETIC HEMATITES}

Hematites weresynthesized with different degrees of isomorphic substitution (IS) of $\mathrm{Fe}$ for Al to a maximum of $16 \mathrm{Al}$ mol \%. Theminerals werecharacterized by X-ray diffraction, total chemical analysis, extraction of iron and al umi num sol ublein ammonium acid oxal ate

\footnotetext{
(1) Recebido para publicação em junho de 2000 e aprovado em julho de 2001.

(2) Pós-Graduando do Departamento de Agronomia, Universidade Estadual de Maringá - UEM. Av. Colombo 5790, CEP 87020-900 Maringá (PR).

(3) Departamento de Agronomia, UEM. Bolsista do CNPq. E-mail: acscosta@uem.br

(4) Professor do Departamento de Agronomia, UEM.

(5) Department of Agronomy, The Ohio State University - OSU. EUA.
} 
$\left(\mathrm{Fe}_{0}+\mathrm{Al}_{0}\right)$, determination of the anion exchange capacity (AEC) and specific surface area (SSA). The results showed that the synthesis methods resulted in a mi nimum amount of impurities and that, in a general way, the foreseen values of isomorphic substitution were achieved. TheSSA values were relatively low for the hematites with up to $10 \%$ of $\mathrm{Al}$, and elevated for thehematites with 13 and $16 \%$ of Al of isomorphic substitution. A good correlation was observed between the SSA and the AEC with the amounts of $\mathrm{Fe}$ and $\mathrm{Al}$, soluble in ammonium acid oxalate $\left(\mathrm{Fe}_{\mathrm{o}}+\mathrm{Al}_{\mathrm{o}}\right)$.

Index terms: specific surface area, AEC, XRD, iron oxides.

\section{NTRODUÇÃO}

Os óxidos de Fe são encontrados em grandes quantidades na natureza e, de acordo com Schwertmann \& Taylor (1989), são os mais abundantes óxidos metálicos presentes nos solos, posicionando-se como o segundo grupo de minerais presentes em solos tropicais, altamente intemperizados. Além dos sol os, estes minerais estão presentes em rochas, sedimentos, poei ra e podem ser encontrados desdetamanho coloidal atémacroscópico. Nos sol os, geralmente, ocorrem em partículas muito pequenas, submicroscópicas e, pela sua natureza química ealta área superficial específica, atuam com eficiência na adsorção de ânions inorgânicos (fosfatos, molibdatos e silicatos), ânions orgânicos (citrato, ácidos húmicos e ácidos fúlvicos), vários biocidas e cátions ( $\mathrm{Co}, \mathrm{Cr}, \mathrm{Cu}, \mathrm{Ni}, \mathrm{Pb}, \mathrm{V}, \mathrm{eZn}$ ) (Kämpf \& Curi, 2000).

Além disso, estes minerais influem na estrutura do solo, geralmente, provocando a formação de agr egados e a cimentação de outros componentes do solo, conferindo a muitos Latossolos brasileiros características físicas específicas que incluem alta porosidade, baixa densidade aparente e uma estrutura granular estável, de forma que estes agregados podem comportar-se, do ponto de vista físico, como partículas de areia (Fontes, 1992).

Segundo Schwertmann \& Taylor (1989), estes minerais estão presentes em muitos solos sob diferentes condições climáticas, em uma ou mais formas e em diferentes níveis de concentração $(0,1$ até $50 \%$ ). Em Latossol os brasileiros, estes minerais ocorrem em concentrações que variam de 0,7 até 44\% (Kämpf et al., 1988; Kämpf \& Curi, 2000). Podem ser minerais primários, como a magnetita $\left(\mathrm{Fe}_{3} \mathrm{O}_{4}\right)$, encontrada, com freqüência, na fração silte e areia de alguns solos, ou, ainda, minerais secundários, resultado do intemperismo de minerais primários ricos em Fe. Nestes casos, o Fe é transformado da forma divalente (Fell) para a forma trivalente (FellI), conferindo aos solos cores que variam do amarel o ao vermel ho (Schwertmann, 1991).

Considerando a alta energia de cristalização e vários efeitos ini bitórios da solução do sol o, os cristais de óxidos de Fe são extremamente pequenos (5$150 \mathrm{~nm}$ ), e desordens nestes cristais são bastante comuns, resultando em el evadas áreas superficiais específicas (ASE), contribuindo, efetivamente, na ASE dos solos (Schwertmann, 1991). Bigham et al. (1978) encontraram val ores de 60 até $200 \mathrm{~m}^{2} \mathrm{~g}^{-1}$ para os óxidos de Fe dos solos estudados e constataram um decréscimo considerável na ASE da fraçãoargila deles, quando os óxidos de Fe foram extraídos com uma solução aquecida de ditionito-citratobicarbonato (Mehra \& J ackson, 1960).

A substituição isomórfica (SI) do Fe pelo Al ou, em menor escala, por outros metais na grade cristalina dos óxidos de Fe, depende basicamenteda disponibilidade destes durante o processo de formação (Schwertmann \& Cornell, 1991). Em condições normais, isto ocorre com freqüência, porque, geralmente, há disponibilidade de AI no sistema (Schwertmann et al., 2000), sendo possível o átomo de Al apresentar a mesma valência e tamanho similares aos do Fe $(r=0,053 \mathrm{~nm}$ para o $\mathrm{Al}^{3+}$ e $\mathrm{r}=0,064 \mathrm{~nm}$ para o $\left.\mathrm{Fe}^{3+}\right)$, podendo, então, substituí-lo na posição octaedral nos óxidos de Fe.

A hematita, segundo óxi do de Fe mais abundante na natureza, é responsável pela coloração avermel hada dos solos. Forma uma solução sólida incompleta com o corundum $\left(\alpha-\mathrm{Al}_{2} \mathrm{O}_{3}\right)$ e substituições de até aproximadamente $16 \%$ mol de Al têm sido observadas, tanto para hematitas sintéticas, quanto para naturais (Peña \& Torrent, 1984; Schwertmann \& Taylor, 1989; Fontes \& Weed, 1991; Schwertmann \& Cornell, 1991; Reis et al., 1995; Costa et al., 1999).

Em Latossol os brasileiros, têm sido encontradas substituições de Fe por Al, em hematitas, entre 4 e $17 \%$ (Kämpf et al., 1988). Uma importante característica da substituição de Fe por AI nos óxidos de Fe é a redução da célula unitária causada pelo menor tamanho do Al (Norrish \& Taylor, 1961). Baseado neste princípio, Schwertmann et al. (1979) determinaram a quantidade deAl substituindo o Fe em hematitas sintetizadas a $70^{\circ} \mathrm{C}$, através da correlação entreo eixo "a" da cela unitária, obtido pelos espaçamentos dos refl exos equivalentes ao plano 110 ou do plano 300: $\mathrm{Al}(\mathrm{mol} \%)=3076,8-610,7 \mathrm{a}$; em que $a=d 110 \times 2$, ou $a=d 300 \times 3,464$, respectivamente.

O presente trabalho teve por objetivo sintetizar hematitas com crescentes graus de SI de Fe por Al, confirmar o grau de substituição efetivamente al cançado por métodos dir eto e indireto e avaliar a 
influência da SI sobre algumas relevantes características químicas e mineralógicas.

\section{MATERIAL E MÉTODOS}

Foram sintetizadas amostras de hematita, com o objetivo de obter essa espécie mineral com crescentes graus de SI de Fe por Al. A hematita pura foi preparada através da transformação da ferridrita, conforme método descrito por Schwertmann \& Cornell (1991); $40 \mathrm{~g}$ de $\mathrm{Fe}\left(\mathrm{NO}_{3}\right)_{3} \cdot 9 \mathrm{H}_{2} \mathrm{O}$ foram dissolvidos em $500 \mathrm{~mL}$ de água destilada e deionizada pré-aquecida a $90^{\circ} \mathrm{C}$, após foram adicionados $300 \mathrm{~mL}$ desol ução $\mathrm{KOH}$ M, também préaquecidos a $90^{\circ} \mathrm{C}$ de modo a precipitar a ferridrita. $\mathrm{Em}$ seguida, foram adicionados $50 \mathrm{~mL}$ de $\mathrm{NaHCO}_{3}$ $\mathrm{M}$ pré-aquecido a $90^{\circ} \mathrm{C}$ e o precipitado volumoso e castanho formado foi colocado em suspensão e acondicionado em frascos de polietileno à temperatura de $90^{\circ} \mathrm{C}$ por 48 horas. As hematitas, com diferentes graus de SI de Fe por Al, foram obtidas através da combi nação de diferentes vol umes de $\mathrm{Fe}\left(\mathrm{NO}_{3}\right)_{3} 0,1 \mathrm{M}$ e $\mathrm{Al}\left(\mathrm{NO}_{3}\right)_{3}$ 0,1M dentro de béqueres de polietileno (Quadro 1). Em seguida, foi adicionado suficiente $\mathrm{NaOH} \mathrm{M}$ para levar o $\mathrm{pH}$ a 7 . O precipitado formado foi lavado por três vezes com água destilada e o $\mathrm{pH}$ corrigido para 7 . Os béqueres foram então armazenados a $80^{\circ} \mathrm{C}$ por 65 dias e, após a cristal ização dos minerais, estes foram lavados com água destilada e dei onizada e secos a $105^{\circ} \mathrm{C}$.

Dissolução total (DT): Todos os minerais foram submetidos à DT. Amostras em duplicata, com aproximadamente $100 \mathrm{mg}$ de mineral, foram colocadas em tubos de ensaio. Em seguida, foram adicionados a cada tubo $15 \mathrm{~mL}$ de $_{2} \mathrm{SO}_{4}$ a $20 \% \mathrm{v} / \mathrm{v}$. Os tubos foram tampados para que não ocorresse evaporação. Elevou-se a temperatura, através de banho-maria, até $80^{\circ} \mathrm{C}$ para acelerar a dissolução. Completada a dissolução de todos os minerais, as soluções foram armazenadas em frascos de polietileno. As concentrações de $\mathrm{Fe}$ e Al foram obtidas através de leitura em espectrofotômetro de absorção atômica, utilizando-se ar-acetileno para o Fe e óxido nitroso para oAl.

Quadro 1. Volume das soluções que continham Fe e Al para a síntese de hematitas com diferentes graus de substituição isomórfica

\begin{tabular}{|c|c|c|}
\hline Mineral & $\mathrm{Fe}\left(\mathrm{NO}_{3}\right)_{3}$ & $\mathrm{Al}\left(\mathrm{NO}_{3}\right)_{3}$ \\
\hline & \multicolumn{2}{|c|}{$\mathrm{mL}$} \\
\hline Hematita $5 \% \mathrm{Al}$ & 1.900 & 100 \\
\hline Hematita $10 \% \mathrm{Al}$ & 1.800 & 200 \\
\hline Hematita $15 \% \mathrm{Al}$ & 1.700 & 300 \\
\hline Hematita $20 \% \mathrm{Al}$ & 1.600 & 400 \\
\hline
\end{tabular}

F ormas de baixa cristalinidade $\left(\mathrm{Fe}_{\mathrm{o}}+\mathrm{Al}_{\mathrm{o}}\right)$ : As formas de Fe e Al de baixa cristalinidade foram determinadas pela uti lização do ataque com oxalato ácido de amônio, conforme descrito em Camargo et al. (1986). Duplicatas com 100 mg de cada mineral foram colocadas em tubos de centrífuga e envoltas em papel-al umínio, de modo a garantir quea solução permanecesse protegida da exposição à luz. Adicionaram-se então $20 \mathrm{~mL}$ do reagente deTamm (solução de oxal ato ácido de amônio) e a mistura foi agitada em mesa agitadora, por 4 h, a 60 oscilações por minuto, seguindo-se de centrifugação a $1.000 \mathrm{rpm}$ por $10 \mathrm{~min}$. A solução sobrenadante foi armazenada em frascos de polietileno para a deter minação das concentrações deFeeAl por meio de leitura em espectrofotômetro de absorção atômica, bem como feito o cálculo das quantidades de $\mathrm{Fe}_{\mathrm{o}} \mathrm{e}$ $\mathrm{Al}_{\mathrm{o}}$ para cada amostra.

Área superficial específica (ASE): Utilizouse, para a determinação da ASE, o método baseado na retenção de líqui dos polares, em quea quantidade adsorvida é proporcional à área das partículas (Dyal \& Hendricks, 1950; E MBRAPA, 1997). Amostras de aproximadamente $500 \mathrm{mg}$, em duplicata, foram colocadas em copos confeccionados em papelalumínio e, em seguida, receberam de 1 a $2 \mathrm{~mL}$ de etileno glicol mono-etil éter (EGME), para se obter a saturação em todos os minerais, e colocadas em dessecador com $\mathrm{CaCl}_{2}$ anidro. Em seguida, aplicouse vácuo ( $0,6 \mathrm{~atm} / 12 \mathrm{~h})$ e foram realizadas pesagens sucessivas atéà obtenção de peso constante. A ASE foi cal culada para cada amostra por meio da seguinte fórmula: ASE $\left(\mathrm{m}^{2} \mathrm{~g}^{-1}\right)=a /(0,000286 \mathrm{~b})$; em que a é a massa de EGME adsorvido pela amostra e b é a massa da amostra seca a $105^{\circ} \mathrm{C}$.

Capacidade de troca aniônica (CTA): A CTA foi obtida de um extrator mecânico seqüencial, utilizando o $\mathrm{Cl}^{-}$como ânion-índice, segundo procedimento descrito em J aynes \& Bigham (1986). Amostras com aproximadamente $500 \mathrm{mg}$ de cada mineral, em duplicata, foram depositadas em seringas de $60 \mathrm{~mL}$, que continham $1 \mathrm{~g}$ de polpa de celulose prensada. Em seguida, montou-se oextrator de modo a possibilitar sucessivas extrações: (1.) Saturação dos sítios de adsorção aniônica dos minerais com $\mathrm{Cl}^{-}$, através da passagem de $50 \mathrm{~mL}$ de $\mathrm{CaCl}_{2} \mathrm{M}$ em uma extração, com duração de 8 h. (2.) Lavagem do excesso de $\mathrm{Cl}^{-}$, através da passagem de $50 \mathrm{~mL}$ de $\mathrm{CaCl}_{2}$ 0,01 M em uma extração de $1 \mathrm{~h}$ de duração (3.) Retirada do excesso de solução que continha $\mathrm{Cl}^{-}$, através de uma lavagem com álcool etílico em uma extração com duração de 1 h. (4.) Remoção de todo $\mathrm{Cl}^{\prime}$ adsorvido pelos minerais, através da passagem de $50 \mathrm{~mL}$ de $\mathrm{KNO}_{3} \mathrm{M}$ em uma extração com $8 \mathrm{~h}$ de duração. Esta última solução foi acondicionada em frascos de polietileno, pesada e determinou-se a quantidade de $\mathrm{Cl}^{-}$através de titulação com nitrato de prata, na presença de cromato de potássio como indicador (Malavolta et al., 1997). Deposse das quantidades de $\mathrm{Cl}^{-}$adsorvido 
por cada mineral, calculou-sea CTA de cada material $\mathrm{em} \mathrm{Cmol}_{\mathrm{C}} \mathrm{kg}^{-1}$ de mineral.

Difratometria de Raios-X (DRX): Todos os minerais sintetizados foram analisados por DRX (Whitting, 1965), tendo si do estas anál ises real izadas nas amostras sem prévio tratamento e, também nestas mesmas amostras, após tratamento prévio com oxalato ácido de amônio para a remoção de possíveis frações de baixa cristalinidade. Todas as amostras foram utilizadas na forma de pó. Difratogramas de raio- $X$ foram obtidos, utilizando radiação $\mathrm{CuK} \alpha$, em uma varredura escal onada de $0,02{ }^{\circ} 2 \theta$ por $3 s$, entre 15 e $65^{\circ} 2 \theta$, em um equipamento Philips Electronics PW1 1316/90, com goniômetro de amplo espectro, ajustado a uma fenda de compensação dos valores dos ângulos theta, uma fenda de entrada dos raios difratados de $0,2 \mathrm{~mm}$ e um filtro monocromador de grafite. As amostras foram adicionadas ao suporte de alumínio e pressionadas levemente, evitando-se a orientação preferencial das partículas. Os valores de intensidade de difração e dos ângulos $2 \theta$ obtidos foram transferidos a um computador, utilizando software e uma interface Databox (Radix Instruments, versão 1990).

Determinação do grau de SI de Fe por Al: A estimativa da substituição isomórfica de Fe por Al foi feita por meio de dois métodos: (1.) Cálculo da SI , avaliando-se a contração da cela unitária, obtida via DRX, dependendo do grau de substituição existente, originando para o presentetrabal ho dois valores: um, para as amostras sem tratamento, e outro, para as amostras submetidas ao tratamento com oxalato ácido de amônio. Determinou-se a quantidade deAl substituindo o Fe através da posição do reflexo equivalente ao plano 110 (Schwertmann et al., 1979). A posição dos reflexos equivalentes aos três planos mais intensos do silício, adicionado como padrão interno ( $5 \% \mathrm{~g} / \mathrm{g})$, em cada amostra, foi utilizada para corrigir os reflexos equivalentes aos planos dos minerais do presente estudo, eliminando possíveis desvios decorrentes do equi pamento e garantindo a apl icabilidade da fórmula para o cál culo da SI (Costa et al., 1999). (2.) Determinação direta, através da DT e um segundo valor, referente apenas ao material cristalino, foi obtida por diferença ponderada entre os val ores de DT e a dissol ução sel etiva com oxalato ácido de amônio (material total - material amorfo = material cristalino). Portanto, nototal, foram obtidas quatro estimativas diretas da SI, sendo duas para os materiais em sua forma original (sem tratamento prévio com oxalato ácido de amônio) e duas para a parte cristalina dos materiais.

\section{RESULTADOS E DISCUSSÃO}

Substituição isomórfica: Foi possível verificar (Quadro 2) que, para a hematita pura, hematita $5 \% \mathrm{Al}$ e hematita $10 \% \mathrm{Al}$, o grau de SI calculado a partir da dissolução total eo cal culado por diferença para o material cristalino foram muito próximos do grau de SI pretendido para estes minerais. Para a hematita denominada hematita $15 \% \mathrm{Al}$, o nível de SI encontrado foi pouco inferior ao pretendido, ao redor dos $13 \%(13,2 \%$, no material total, e $12,8 \%$, no material cristalino). J á para a hematita de $20 \% \mathrm{Al}$, valores ao redor dos $16 \%$ foram determinados (15,7\%, para o material total, e $16,6 \%$, para o material cristalino), permanecendo, praticamente, no limite máximo, de $16 \% \mathrm{Al}$ mol , encontrado por vários autores (Peña \& Torrent, 1984; Schwertmann \& Taylor, 1989; Fontes \& Weed, 1991; Schwertmann \& Cornell, 1991; Reis et al., 1995, Costa et al., 1999), no qual $1 / 6$ dos átomos de Fe foi substituído por átomos de Al.

Os valores de SI encontrados para os materiais cristalinos detodas as hematitas foram praticamente iguais aos valores de SI encontrados para os materiais totais dessas hematitas. Tais resultados são decorrentes da menor quantidade de material de baixo grau de cristalinidade determinado para a hematita pura, hematita 5\% Al e hematita $10 \% \mathrm{Al}$, minimizando o efeito do teor dessa fração sobre a SI do material cristalino, remanescente desses minerais.

Quadro 2. Características químicas e mineralógicas das hematitas com diferentes graus de substituição isomórfica de Fe por Al (médias de duas repetições).

\begin{tabular}{|c|c|c|c|c|c|c|c|}
\hline \multirow{2}{*}{ Mineral } & \multicolumn{4}{|c|}{ SI (dissolução total) } & \multirow[b]{2}{*}{$\mathrm{Fe}_{\mathrm{o}}+\mathrm{Al}_{\mathrm{o}}$} & \multirow[b]{2}{*}{ ASE } & \multirow[b]{2}{*}{ CTA } \\
\hline & Total & Cristalino & Total & Cristalino & & & \\
\hline & & & & & dag kg-1 & $\mathrm{m}^{2} \mathrm{~g}^{-1}$ & $\mathrm{cmol}_{\mathrm{c}} \mathrm{kg}^{-1}$ \\
\hline Hematita pura & 0,2 & 0,2 & 0,6 & 1,3 & 0,9 & 10,1 & 3,6 \\
\hline Hematita $5 \% \mathrm{Al}$ & 5,5 & 5,4 & 5,2 & 3,9 & 0,3 & 8,8 & 7 \\
\hline Hematita $10 \% \mathrm{Al}$ & 9,8 & 9,7 & 12,3 & 8,9 & 0,9 & 17,8 & 6,6 \\
\hline Hematita $15 \% \mathrm{Al}$ & 13,2 & 12,8 & 12,4 & 15,8 & 21,4 & 56,8 & 10,5 \\
\hline Hematita $20 \% \mathrm{Al}$ & 15,7 & 16,6 & 20,02 & 17,6 & 47,7 & 137,9 & 32,8 \\
\hline
\end{tabular}



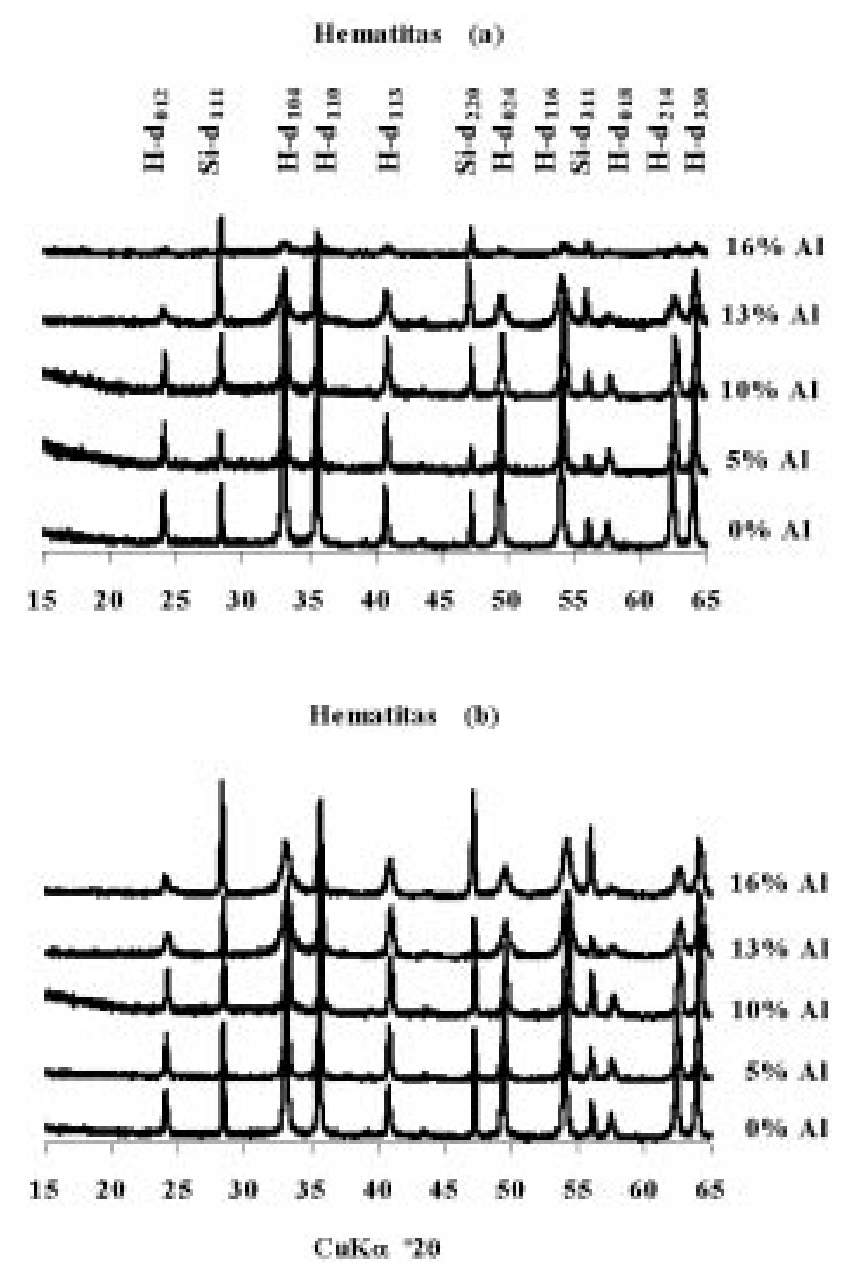

Figura 1. Difratogramas de raio-X (DRX) para as hematitas $(\mathrm{H})$ com adição de silício (Si), antes (a) depois (b) do tratamento com oxalato ácido de amônio.

As hematitas $15 \% \mathrm{Al}$ e $20 \% \mathrm{Al}$ sintetizadas apresentaram graus de SI diferentes daqueles pretendidos, razão por que foram renomeadas para hematita $13 \%$ Al ehematita $16 \% \mathrm{Al}$, respectivamente, para representarem os verdadeiros teores de Al presentes, conforme determinação via dissolução total. A equação proposta por Schwertmann et al. (1979), utilizando-se da posição do reflexo do plano 110 (Figura 1), gerou resultados bastante próximos daqueles obtidos (Quadro 2) através da dissol ução total, apesar de ser um método de mensuração indireta, evidenciando a eficiência da equação utilizada na avaliação do grau de SI. Esta mesma equação foi considerada, por Kämpf \& Schwertmann (1998), a mais adequada para avaliar o grau de SI de Fe por Al em hematitas aluminosas de solos.

Dentre os desvios existentes entre esta determinação e a dissolução total, percebeu-se uma predominância de desvios superiores, gerando indícios de que, para este grupo de hematitas, a equação utilizada gerou valores de SI ligeiramente superiores. Ainda, pode-se notar (Quadro 2) que, para um mesmo mineral, foram determinados val ores de SI um pouco diferentes entre o material total e o material residual, após tratamento com oxalatoácido de amônio, sem, contudo, dar indícios de alguma tendência, pois ora foi o material total (hematita $5 \% \mathrm{Al}, 10 \% \mathrm{Al}$, e $16 \% \mathrm{Al}$ ), ora foi o material tratado (hematita pura, e 13\% Al) que apresentaram os maiores valores de SI. Na utilização deste método, devem-seconsiderar pequenas variações nas posições dos reflexos equivalentes aos planos que resultam em consi deráveis variações na SI calculada, uma vez que mínimos desvios na sua identificação geram desvios consi deráveis no valor da SI calculada, mesmo utilizando a adição de silício como padrão interno.

Correlação significativa $(R=0,93)$ foi obtida entre o grau de SI determinado para o material total e o grau deSI referente ao material cristalino, para as hematitas do presente estudo, que, assi m como obtido através da dissolução total, apresentou valores de SI muito próximos para as duas fases.

Formas de baixa cristalinidade: Dentre as hematitas desteestudo, determinaram-se quantidades de $\mathrm{Fe}$ e Al solúveis em oxalato ácido de amônio bastante variadas (Quadro 2). I sto é, as hematitas com menor grau deSI, até $10 \%$, apresentaram baixos teores de FeeAl solúveis em oxalatoácido de amônio, já a hematita $13 \% \mathrm{Al}$ apresentou 21,4 dag $\mathrm{kg}^{-1}$ de $\mathrm{Fe}_{0}+\mathrm{Al}_{0}$, enquanto a hematita $16 \% \mathrm{Al}$ apresentou 47,7 dag $\mathrm{kg}^{-1}$.

A el evada quantidade de $\mathrm{Fe}_{\mathrm{o}}+\mathrm{Al}_{\mathrm{o}}$ encontrada para estas duas hematitas determinou que a presença do Al em maiores concentrações, nas soluções, inibiu a formação de minerais com cristais desenvolvidos. Tal fato pode ser confirmado quando se observam os difratogramas de raio-X (Figura 1 ). Os DRX evidenciam a não-existência de diferenças entre o material total e o material residual após tratamento com oxalato ácido de amônio para as três primeiras hematitas, porém, para as hematitas $13 \% \mathrm{Al}$ e $16 \% \mathrm{Al}$, facilmente visual iza-sea diferença entre os difratogramas dos minerais antes e depois do tratamento, indicando que a fração de baixo grau de cristalinidade foi removida, permitindo melhor visual ização dos reflexos equivalentes dos planos da fração cristalina.

Mesmo para a fração tratada, foi evidenciada a presença de material com cristalização pouco desenvolvida, apesar de resistente à dissolução seletiva a que foram submetidos, já que, para estes minerais, a largura à meia-altura mostrou-se muito maior que para as três primeiras hematitas. Contudo, esta grande quantidade de material removido através da dissol ução sel etiva não se trata, pel o menos em sua maior parte, de ferridrita, queé, segundoSchwertmann \& Taylor (1989) eSchwertmann (1991), um óxido de ferro completamente solúvel em oxalato áci do de amônio, já que não se observou nos difratogramas seu comportamento característico. 
O material removido com oxalatoáci do deamônio nas hematitas $13 \% \mathrm{Al}$ e $16 \% \mathrm{Al}$ aparentemente trata-se dehematita, mas com grau decristalinidade muito reduzido e porosidade muito alta, passível de dissolução (Torrent et al., 1987; Schwertmann, 1991), apesar de terem permanecido, como as demais, por um longo período de "cura", 65 dias a $80^{\circ} \mathrm{C}$, período, insuficiente para seu desenvolvimento e definição de maior grau de cristalinidade.

Gamble \& Daniels (1972) determinaram razão Fe solúvel em oxalato ácido de amônio/Fe solúvel em ditionito $\left(\mathrm{Fe}_{\mathrm{d}} / \mathrm{Fe}_{\mathrm{d}}\right)$ da ordem de até 0,20 para duas hematitas comerciais. Ainda, Torrent et al. (1987) sintetizaram uma série de hematitas por diferentes métodos e, após uma lavagem do material final com oxalato áci do de amônio seguida de várias lavagens com $\left(\mathrm{NH}_{4}\right)_{2} \mathrm{CO}_{3} \mathrm{M}$ e secagem a $70^{\circ} \mathrm{C}$ para remoção de todo material amorfo, obtiveram razão $\mathrm{Fe}_{d} / \mathrm{Fe}_{\mathrm{d}}$ de até0,31. Torrent et al. (1987) observaram que, embora a quantidade de oxalato ácido de amônio utilizada na lavagem dos materiais fosse várias vezes maior do quea quantidade estequi ométrica necessária para a remoção dos materiais amorfos, o material resultanteainda teria al gum "material amorfo", não confirmado pel os autores.

Os difratogramas diferenciais de raio- $X$ realizados, subtraindo os difratogramas das amostras de hematitas tratadas e não tratadas com oxalato ácido de amônio (dados não apresentados), confirmam estas observações; não apresentaram os seis reflexos equivalentes aos planos difusos de difração da ferridrita, como observado por Schwertmann et al. (1982) na identificação deste mineral em amostras de sol os gl eizados, mesmo na amostra de hematita $16 \% \mathrm{Al}$, que apresentou 47,7 dag kg-1 de $\mathrm{Fe}_{\mathrm{o}}+\mathrm{Al}_{\mathrm{O}}$.

Área superficial específica (ASE): A ASE, determinada via adsorção do EGME, apresentou grande variação dentre as hematitas do estudo, com valores variando de 10 a mais de $100 \mathrm{~m}^{2} \mathrm{~g}^{-1}$ (Quadro 2). A hematita pura e a hematita 5\% Al apresentaram valores reduzidos de ASE, 10,1 e $8,8 \mathrm{~m}^{2} \mathrm{~g}^{-1}$, respectivamente. Estes valores foram muito próximos aos menores valores determinados por Barrón et al. (1988), para hematitas sintéticas, e por Torrent et al. (1994), para hematitas naturais, quando da utilização de $\mathrm{N}_{2}$ como substância adsorvida. Para a hematita $10 \% \mathrm{Al}$, foi obtido $17,8 \mathrm{~m}^{2} \mathrm{~g}^{-1} \mathrm{e}$, apesar de ser significativamente superior aos minerais anteriores, ainda se trata de uma ASE relativamente baixa, revelando, para estas três hematitas, al to grau de cristalização.

As hematitas $13 \mathrm{Al}$ e $16 \% \mathrm{Al}$ apresentaram valores de ASE muito superiores aos das três anteriores: 56,8 e 137,9 $\mathrm{m}^{2} \mathrm{~g}^{-1}$, respectivamente. Estes dois minerais mostraram altos teores de $\mathrm{Fe}_{\mathrm{o}}+\mathrm{Al}_{0}$, evidenciando a presença de grandes quantidades de óxidos de Fe com baixo grau de cristalinidade. Considerando a ASE apresentada, poderia tratar-se de ferridrita, que, segundo Schwertmann \& Taylor (1989), apresentou valores deASE de 200 a $500 \mathrm{~m}^{2} \mathrm{~g}^{-1}$; porém, estes resultados foram muito próximos dos obtidos por Barrón et al. (1988), para hematitas microcristalinas, para as quais determinaram valores de até $115 \mathrm{~m}^{2} \mathrm{~g}^{-1}$, obtidos através de adsorção de $\mathrm{N}_{2}$, que, geral mente, é menor do queASE determinada pela adsorção de EGME.

Torrent et al . (1987) encontraram valores deASE para hematitas sintéticas, mesmo após a remoção do material amorfo com oxalato ácido de amônio, que variaram de 6 a $138 \mathrm{~m}^{2} \mathrm{~g}^{-1}$, onde os maiores val ores foram observados naquel as queapresentaram o maior grau de substituição isomórfica e relação Feo/Fed. Torrent et al. (1987) afirmaram que estes valores elevados de ASE podem estar associados a diferenças na morfologia dos cristais, irregularidades na sua superfície ou à presença de microporos. Schwertmann et al. (2000) também encontraram para hematitas sintetizadas a $25^{\circ} \mathrm{C}$ ASE, relativamente pequenas (até $20 \mathrm{~m}^{2} \mathrm{~g}^{-1}$ ) com até $10 \%$ de substituição isomórfica; acima desseval or deSI , observaram um grande acréscimo na ASE mensurada pela adsorção de $\mathrm{N}_{2}$, al cançando $57 \mathrm{~m}^{2} \mathrm{~g}^{-1}$. Esses autores observaram que o acentuado aumento na ASE correlacionou-se, estreitamente, com o volume de microporos existente nestas hematitas. Tais resultados vêm corroborar a hi pótese de que, apesar de solúvel em oxalato ácido de amônio, estas hematitas são microcristalinas (Barrón et al., 1988), o que explicaria os el evados valores de ASE, e que as duas hematitas, que apresentaram el evadas ASEs, continham grandes volumes de microporos (Torrent et al., 1987). Obteve-se, então, uma alta correlação positiva $(R=0,99)$, entre ASE e os teores de $\mathrm{Fe}_{0}+\mathrm{Al}_{\mathrm{O}}$, representada pela seguinte equação linear: $\mathrm{ASE}=$ $8,8759+2,63\left(\mathrm{Fe}_{\mathrm{o}}+\mathrm{Al}_{\mathrm{o}}\right)$, revelando a estreita correlação entreograu deSI ea ASE para estegrupo de hematitas.

Capacidade de troca aniônica: A CTA determinada para as hematitas mostrou uma ampla variação, desde $3,6 \mathrm{cmol}_{\mathrm{c}} \mathrm{kg}^{-1}$, referente à hematita pura, até $32,8 \mathrm{cmol}_{\mathrm{c}} \mathrm{kg}^{-1}$, apresentando um comportamento crescente, com o acréscimo do grau deSI deFepor Al, teor de $\mathrm{Fe}_{0}+\mathrm{Al}_{0}$ ea ASE. Valores de CTA para minerais puros foram raramente citados na literatura. Para sol os tropicais, Li (1995) apresentou val ores de CTA menores que $1 \mathrm{cmol}_{\mathrm{c}} \mathrm{kg}^{-1}$, variando tais valores conforme os teores dos óxidos de Fe encontrados nos solos.

A determinação da CTA em $\mathrm{cmol}_{\mathrm{c}} \mathrm{kg}^{-1}$ revel ou a quantidade de cargas superficiais positivas existentes por unidade de massa, responsáveis pela retenção do $\mathrm{Cl}^{-}$, bem como do EGME utilizado na medida da área superficial. Os altos coeficientes de correlação obtidos entre os valores de CTA e os valores de $\mathrm{SI}(\mathrm{R}=0,89), \mathrm{Fe}_{0}+\mathrm{Al}_{\mathrm{o}}(\mathrm{R}=0,96)$ e ASE $(R=0,98)$ demonstraram a correlação entre as 
características químicas e mineralógicas e a estrutura cristalina e morfológica determinadas pela substituição isomórfica de Fe pelo Al. Como a equação que representou o comportamento das hematitas em relação a essas duas características foi linear, concluiu-se que, para todas elas, a quantidade de cargas positivas por unidade de área foi praticamente a mesma, isto é: $2,106 \cdot 10^{-4} \mathrm{cmol}_{\mathrm{c}} \mathrm{m}^{-2}$.

\section{CONCLUSÕES}

1. A utilização de minerais sintetizados em condições de laboratório foram excel entes meios de avaliar a capacidade de reação de diferentes hematitas.

2. Os baixos níveis de substituição isomórfica de Fepor Al não provocaram expressivas alterações nas características químicas e mineralógicas das hematitas avaliadas.

3. Níveis elevados de SI, próximos ao máximo reconhecidamente admitido de $16 \mathrm{Al} \mathrm{mol} \%$, provocaram a formação de cristais de dimensões reduzi das, implicando a existência de el evadas ASEs e CTA.

\section{LITE RATURA CITADA}

BARRÓN, V.; HERRUZO, M. \& TORRENT, J. Phosphate adsorption by aluminous hematites of different shapes. Soil Sci. Soc. Am. J., 52:647-651, 1988.

BIGHAM, J.M.; GOLDEN, D.C.; BUOL, S.W.; WEED, S.B. \& BOWEN, L.H. I ron oxide mineralogy of well-drained UItisols and Oxisols: II. Influence on color, surface area, and phosphate retention. Soil Sci. Soc. Am. J., 42:825830, 1978.

CAMARGO, O.A.; MONIZ, A.C.; J ORGE, J .A. \& VALADARES, J.M.A.S. Métodos de análise química, mineralógica efísica de solos do Instituto Agronômico de Campinas. Campinas, Instituto Agronômico de Campinas, 1986. 94p. (Boletim Técnico, 106)

COSTA, A.C.S.; BIGHAM, J .M.; RHOTON, F.E.; \& TRAINA, S.J . Quantification and characterization of maghemite in soils derived from volcanic rocks in southern Brazil. Clays Clays Miner., 47:466-473, 1999.

DYAL, R.S. \& HENDRICKS, S.B. Total surface of clays in polar liquids as a characteristic index. Soil Sci., 69:421-432, 1950.

EMPRESA BRASILEIRA DE PESQUISA AGROPECUÁRIA EMBRAPA. CentroNacional de Pesquisa de Solos. Manual de métodos de análises de solo. 2.ed. Rio de J aneiro, 1997. $212 p$.

FONTES, M.P.F. I ron oxide-clay mineral association in brazilian oxisols: A magnetic separation study. Clays Clay Miner., 40:175-179, 1992.
FONTES, M.P.F. \& WEED, S.B. I ron oxides in selected Brazilian Oxisols: I. Mineralogy. Soil Sci. Soc. Am. J., 55:11431149, 1991.

GAMBLE, E.E. \& DANIELS, R.B. I ron and silica in water, acid ammonium oxalate, and dithionite extracts of some North Carolina Coastal plain soils. Soil Sci. Soc. Am. Proc., 36:939-943, 1972.

J AYNES, W.F. \& BIGHAM,J.M. Multiplecation-exchange capacity measurements on standard clays using a commercial mechanical extractor. Clays Clay Miner., 34:93-98, 1986.

KÄMPF, N. \& CURI, N. Óxidos deferro: indicadores deambientes pedogênicos e geoquímicos, In: NOVAIS, R.F.; ALVAREZV., V.H. \& SCHAEFER, C.E.G.R, eds. Tópicos em ciência do solo. Viçosa, Sociedade Brasileira de Ciência do Solo, 2000. p.107-138.

KÄMPF, N.; RESENDE, M. \& CURI, N. I ron oxides in Brazilian Oxisols. In: BEINROTH, F.H.; CAMARGO, M.N. \& ESWARAN, H., eds. INTERNATIONAL SOIL CLASSIFICATION WORKSHOP, 8., Rio de J aneiro, 1988. Proceedings. Rio deJ aneiro, EMBRAPA-SNLCS/USDA/UPR, 1988. p.7177.

KÄMPF, N. \& SCHWERTMANN, U. Avaliação da estimativa de substituição de Fe por Al em hematitas de solos. R. Bras. Ci. Solo, 22:209-213, 1998.

LI, G.L. Eletrostatic adsorption of anions. In:YU,T.R. Chemistry of variable charge soils. New York, Oxford U niversity Press, 1995. p.112-139.

MALAVOLTA, E.; VITTI, G.C. \& OLIVEIRA, S.A. Avaliação do estado nutricional das plantas: princípios e aplicações. Piracicaba, Potafos, 1997.

MEHRA, O.P. \& J ACKSON, M.L. I ron oxide removal from soils by a dithionite-citrate system buffered with sodium bicarbonate. In: SWINEFORD, A., ed. NATIONAL CLAY CONFERENCE, 7., New York, 1958. Proceedings. New York, Pergamon Press, 1960. p.317-327.

NORRISH, K. \& TAYLOR, R.M. The isomorphous replacement of iron by aluminum in soil goethites. J. Soil Sci., 12:294306, 1961.

PEÑA, F. \& TORRENT, J. Relationships between phosphate sortion and iron oxides in alfisols from a river terrace sequence of Mediterranean Spain. Geoderma., 33:283-296, 1984.

REIS, C.; FABRIS, J.D.; NOVAIS, R.F.; BAHIA FILHO, A.F.C.; SANTANA, D.P.; CURI, N. \& COEY, J.M.D. Cinética de sorção de fósforo em al guns sol os de Minas Gerais. R. Bras. Ci. Solo, 19:337-344, 1995.

SCHWERTMANN, U. Solubility and dissolution of iron oxides. In: CHEN, Y. \& HADAR, Y., eds. I ron nutrition and interactions in plants. Dordrecht: Kluwer Academic Publishers, 1991. p.3-27.

SCHWERTMANN, U.; FITZPATRICK, R.W.; TAYLOR, R.M. \& LEWIS, D.G. The influence of aluminum substitution on iron oxides: 2. Preparation and properties of Al-substituted hematites. Clays Clay Miner., 27:105-122, 1979.

SCHWERTMANN, U.; SCHULZE, D.G. \& MURAD, E. I dentification of ferrihidrite in soils by dissolution kinetics, differential X-ray diffraction, and mossbauer spectroscopy. Soil Sci. Soc. Am. J ., 46:869-875, 1982. 
SCHWERTMANN, U. \& TAYLOR, R.M. I ron Oxides. In: DIXON, J.B. \& WEED, S.B.,eds. Minerals in soil environments. Madison, Soil Science Society of America Book Series, 1989. p.379-438.

SCHWERTMANN, U. \& CORNELL, R.M. Iron oxides in the laboratory. New York, Cambridge, VCH, 1991.137p.

SCHWERTMANN, U.; FRIEDL, J .; STANJ EK, H. \& SCHULZE, D.G. The effect of AI on Fe oxides. XIX. Formation of AI substituted hematite from ferrihydrite at $25^{\circ} \mathrm{C}$ and $\mathrm{pH} 4$ to 7. Clays Clay Miner., 48:159-172, 2000.
TORRENT, J ; SCHWERTMANN, U. \& BARRON, V. The reductive dissolution of synthetic goethite and hematite in dithionite. Clay Miner., 22:329-337, 1987.

TORRENT, J .; SCHWERTMANN, U. \& BARRÓN, V. Phosphate sorption by natural hematites. Eur. J. Soil Sci., 45:45-51, 1994.

WHITTING, L.D. X-ray techniques for mineral identification and mineralogical composition. In: BLACK, C.A., ed. Methods of soil analysis. Part I.. Madison, Soil Science Society of America, 1965. p.671-698. (Monography, 9) 